\title{
Skyrme and Wigner crystals in graphene
}

\author{
R. Côté, ${ }^{1}$ J.-F. Jobidon, ${ }^{1}$ and H. A. Fertig ${ }^{2}$ \\ ${ }^{1}$ Département de physique and RQMP, Université de Sherbrooke, Sherbrooke, Québec, Canada, J1K 2R1 \\ ${ }^{2}$ Department of physics, Indiana University, Bloomington, Indiana, 47405, U.S.A.
}

(Dated: November 9, 2018)

\begin{abstract}
At low-energy, the band structure of graphene can be approximated by two degenerate valleys $\left(K, K^{\prime}\right)$ about which the electronic spectra of the valence and conduction bands have linear dispersion relations. An electronic state in this band spectrum is a linear superposition of states from the $A$ and $B$ sublattices of the honeycomb lattice of graphene. In a quantizing magnetic field, the band spectrum is split into Landau levels with level $N=0$ having zero weight on the $B(A)$ sublattice for the $K\left(K^{\prime}\right)$ valley. Treating the valley index as a pseudospin and assuming the real spins to be fully polarized, we compute the energy of Wigner and Skyrme crystals in the Hartree-Fock approximation. We show that Skyrme crystals have lower energy than Wigner crystals i.e. crystals with no pseudospin texture in some range of filling factor $\nu$ around integer fillings. The collective mode spectrum of the valley-skyrmion crystal has three linearly-dispersing Goldstone modes in addition to the usual phonon mode while a Wigner crystal has only one extra Goldstone mode with a quadratic dispersion. We comment on how these modes should be affected by disorder and how, in principle, a microwave absorption experiment could distinguish between Wigner and Skyrme crystals.
\end{abstract}

PACS numbers: 73.20.Qt, 73.21.-b, 73.22.Lp

\section{INTRODUCTION}

For a conventional two-dimensional electron gas (2DEG) created in a semiconductor heterostructure, theoretical calculations show that, in the presence of a strong perpendicular magnetic field, a Wigner crystal (WC) state has lower energy then the fractional quantum Hall liquids for filling factors $\nu \lesssim 1 / 6.5 \underline{\underline{1}}$. Transport measurements indicative of this electron crystallization has been reported by several groups ${ }^{2}$. These measurements include the observation of a strong increase in the diagonal resistivity $\rho_{x x}$, non-linear $I-V$ characteristics, and broadband noise. Another series of experiments involving microwave absorption ${ }^{3}$ have also detected a resonance in the real part of the longitudinal conductivity, $\sigma_{x x}(\omega)$, that has been attributed to the pinning mode of a disordered WC. Such a resonance was observed not only at small filling factor $\nu \lesssim 1 / 6.5$ in the lowest Landau level but also at small filling factor in the higher Landau levels where the formation of a quasiparticle solid is expected in a clean sample. In higher Landau levels, a study of the evolution of the pinning mode with filling factor reveals several transitions of the two-dimensional electron gas ground state from a Wigner crystal at low $\nu$ to a series of bubble crystals with increasing number of electrons per lattice site as $\nu$ is increased, and into a modulated stripe state (or anisotropic Wigner crystal) near half filling $\frac{4}{2}, \frac{5}{.}$.

In a conventional 2DEG, the Landau level energy spectrum is given by $E_{n}=(n+1 / 2) \hbar \omega_{c}$ where $\omega_{c}=e B / m^{*} c$ is the cyclotron frequency with $m^{*}$ the effective mass. Each of these levels is highly degenerate, so that a partially filled Landau level is dominated by electronelectron interactions and is expected to enter a crystal state. Similar physics is expected to occur in graphene.
In a strong perpendicular magnetic field, there is also a series of highly degenerate Landau levels, with energies given by $E_{n}=\operatorname{sgn}(n) \frac{\sqrt{2} \hbar v_{F}}{\ell} \sqrt{|n|}$ where $v_{F}$ is the Fermi velocity. In two recent papers, Zhang and Joglekar $\frac{6}{7}, \frac{7}{7}$ have explored the possibility of Wigner crystallization in graphene (including bubbles and stripes) in the presence of a quantizing magnetic field. While the situations in the presence of a field are similar for the conventional 2DEG and graphene, in the absence of a field they are likely to be different. At low densities, electrons in the former system are believed to form a Wigner crystal. However, graphene in zero field has a gapless non-interacting spectrum, so that an arrangement of electrons into a two-dimensional lattice cannot create effective potentials which localize the individual electrons. This "Klein paradox" physics 8 undermines the stability of the Wigner crystal. In a magnetic field, however, the kinetic energy contains a series of gaps, and the physics of the 2DEG is dominated by the Coulomb interaction alone. A magnetic-field induced Wigner solid is thus expected ${ }^{9}$.

In the Hartree-Fock approximation (HFA), the potential energy depends on the effective Hartree, $H_{n}(\mathbf{q})$, and Fock, $X_{n}(\mathbf{q})$ interactions defined in Landau level $n$. To an excellent approximation $\frac{10}{}$, these effective interactions for $n=0$ are identical to that of a conventional 2DEG so that the HFA phase diagram should be the same in both systems. To develop the analogy further, we use a pseudospin language in which the two non-equivalent valleys $K$ and $K^{\prime}$ of graphene (the valence and conduction bands in graphene touch at two inequivalent points $\mathbf{K}$ and $\mathbf{K}^{\prime}=-\mathbf{K}$ and four other points or valleys related by symmetry) are mapped to the valley pseudospin $| \pm\rangle$ states. If we assume that the real spins are completely polarized in the graphene system, then, for $n=0$, the HF hamiltonian for the 2DEG in graphene is identical to 
that of a conventional 2DEG with zero Zeeman coupling. For $n \neq 0$, however, the effective interactions are not identical. On this basis, we can expect that pseudospin skyrmion crystals are possible in graphene around filling factor $\nu=1$. In a conventional 2DEG, skyrmion crystals are restricted to the lowest Landau level only but this may not be the case in graphene. Indeed, recently Yang, Das Sarma and MacDonald have shown that skyrmions are the lowest-energy charged excitations in graphene for Landau levels up to $n=3^{11}$.

In this paper, we explore the possibility of Skyrmion crystals near integer filling in each Landau level, and compare their stability and collective mode properties to those of Wigner crystal states ${ }^{6,7}$. Because there is no effective Zeeman coupling in graphene, the ground state is generically a crystal of merons rather than of skyrmions, as it is also the case in the conventional $2 \mathrm{DEG}^{12}$. We then compute the collective mode dispersions in the Wigner and meron crystal phases. We show that the approximate $\mathrm{SU}(2)$ symmetry of the hamiltonian leads for a Wigner crystal to a quadratically dispersing valley-pseudospin gapless mode in addition to the phonon mode present in the conventional 2DEG. In the meron crystal, we find instead three new gapless modes with linear dispersions in addition to the phonon mode. In graphene, these modes represent charge fluctuations between the sublattices instead of spin fluctuations as in a conventional 2DEG and so we speculate that they may be visible in microwave absorption experiments. Each crystal structure, Wigner or Skyrme, may thus have a unique signature in microwave absorption, in contrast to Wigner and spin Skyrmion crystals in conventional 2DEG's where the absorption spectrum does not distinguish between these two structures.

This paper is organized in the following way. We review in Secs. II and III some basic properties of graphene and summarize our Hartree-Fock and time-dependent Hartree-Fock formalism for computing the phase diagram and collective excitations. Our numerical results are presented in Sec. IV. We discuss in Sec. V how the collective modes that we find should be affected by the presence of disorder and speculate on their visibility in microwave absorption experiments. We conclude in Sec. VI.

\section{HARTREE-FOCK HAMILTONIAN}

In this section, we briefly explain the Hartree-Fock formalism that we use to compute the energy and collective excitations of the crystal states. We start by reviewing the model hamiltonian for electrons in undoped graphene around the Fermi energy ${ }^{13}$.

A lattice point in graphene is given by $\mathbf{R}=n_{1} \mathbf{a}_{1}+n_{2} \mathbf{a}_{2}$ where $n_{1}, n_{2}$ are positive or negative integers and the primitive vectors are chosen as $\mathbf{a}_{1}=a / 2 \widehat{\mathbf{x}}-\sqrt{3} / 2 \widehat{\mathbf{y}}$ and $\mathbf{a}_{2}=a \widehat{\mathbf{x}}$ with the two carbon atoms in the unit cell at positions $\mathbf{r}_{1}=0$ and $\mathbf{r}_{2}=-c_{0} \widehat{\mathbf{y}}$ where $c_{0}=a / \sqrt{3}=$ $1.42 \AA$ is the separation between two adjacent carbon atoms. We define the carbon atoms with basis vector $\mathbf{r}_{1}$ $\left(\mathbf{r}_{2}\right)$ as part of the $A(B)$ sublattice. The tight-binding hamiltonian for electrons in the $p_{z}$ orbitals of the carbon atoms is given by

$$
H=-t_{0} \sum_{\langle i, j\rangle}\left(a_{i}^{\dagger} b_{j}+\text { h.c. }\right)
$$

where $a_{i}\left(b_{i}\right)$ is the annihilation operator for an electron on the $A(B)$ sublattice of graphene at site $i$ and the summation is over nearest neighbors only with hopping energy (between different sublattices) $t_{0} \approx 2.8 \mathrm{eV}$. In this approximation, the dispersion relations for the valence $(-)$ and conduction $(+)$ bands are given by

$$
\begin{aligned}
E_{ \pm}(\mathbf{k})= & \pm t_{0}\left[1+4 \cos ^{2}\left(\frac{k_{x} a}{2}\right)\right. \\
& \left.+4 \cos \left(\frac{k_{x} a}{2}\right) \cos \left(\frac{\sqrt{3} k_{y} a}{2}\right)\right]^{1 / 2} .
\end{aligned}
$$

For undoped graphene, the Fermi level is at energy $E=$ 0 . With our choice of orientation for the Bravais lattice, the positions of the two non equivalent Dirac points are at $\mathbf{K}=-4 \pi / 3 a \widehat{\mathbf{x}}$ and $\mathbf{K}^{\prime}=4 \pi / 3 a \widehat{\mathbf{x}}$. Around each of these points in $\mathbf{k}$-space, the dispersion of the conduction and valence bands can be approximated by

$$
E(\mathbf{K}+\mathbf{p})=E\left(\mathbf{K}^{\prime}+\mathbf{p}\right) \approx \pm \hbar v_{0}|\mathbf{p}|
$$

where $v_{0}=3 c_{0} t_{0} / 2 \hbar$ is the Fermi velocity. In the $(A, B)$ basis, the hamiltonians around the Dirac points for electrons in the conduction $(+)$ or valence $(-)$ band are given by

$$
H_{ \pm}(\mathbf{p})= \pm \hbar v_{0}\left(\begin{array}{cc}
0 & p e^{ \pm i \theta_{\mathbf{p}}} \\
p e^{\mp i \theta_{\mathbf{p}}} & 0
\end{array}\right)
$$

where $\theta_{\mathbf{p}}$ is the angle between wavevector $\mathbf{p}$ and the $x$ axis.

In the presence of a transverse magnetic field $\mathbf{B}=B_{0} \widehat{\mathbf{z}}$, the hamiltonian is obtained by making the Peierls substitution $E(\mathbf{p}) \rightarrow E(\mathbf{p}+e \mathbf{A} / \hbar c)$, where $\mathbf{A}$ is the vector potential of the magnetic field defined such that $\nabla \times \mathbf{A}=\mathbf{B}$. In terms of the covariant momentum $\mathbf{P}=-i \hbar \nabla+e \mathbf{A} / c=$ $\hbar \mathbf{p}+e \mathbf{A} / c$, we have

$$
H_{ \pm}(\mathbf{p})= \pm v_{0}\left(\begin{array}{cc}
0 & P_{x} \pm i P_{y} \\
P_{x} \mp i P_{y} & 0
\end{array}\right)
$$

with the commutation relation

$$
\left[P_{x}, P_{y}\right]=-i \frac{\hbar^{2}}{\ell^{2}}
$$

where $\ell=\sqrt{\hbar c / e B}$ is the magnetic length. The original conical dispersions at Dirac points $\mathbf{K}$ and $\mathbf{K}^{\prime}$ are now 
split into a set of degenerate Landau levels which have quantized energies given by

$$
E_{n}=\operatorname{sgn}(n) \frac{\sqrt{2} \hbar v_{0}}{\ell} \sqrt{|n|}
$$

where $n=0, \pm 1, \pm 2, \ldots$ The wavefunctions for an electron in these Landau levels (again in the $A, B$ basis) are given by

$$
\begin{aligned}
\left\langle\mathbf{r} \mid \mathbf{K}^{\prime} ; 0, k\right\rangle & =e^{-i \mathbf{K}^{\prime} \cdot \mathbf{r}}\left(\begin{array}{c}
h_{0, k}(\mathbf{r}) \\
0
\end{array}\right), \\
\langle\mathbf{r} \mid \mathbf{K} ; 0, k\rangle & =e^{-i \mathbf{K} \cdot \mathbf{r}}\left(\begin{array}{c}
0 \\
h_{0, k}(\mathbf{r})
\end{array}\right)
\end{aligned}
$$

for Landau level $n=0$, and by

$$
\begin{aligned}
\left\langle\mathbf{r} \mid \mathbf{K}^{\prime} ; n, k\right\rangle & =\frac{1}{\sqrt{2}} e^{-i \mathbf{K}^{\prime} \cdot \mathbf{r}}\left(\begin{array}{c}
h_{|n|, k}(\mathbf{r}) \\
\operatorname{sgn}(n) h_{|n|-1, k}(\mathbf{r})
\end{array}\right)(10) \\
\langle\mathbf{r} \mid \mathbf{K} ; n, k\rangle & =\frac{1}{\sqrt{2}} e^{-i \mathbf{K} \cdot \mathbf{r}}\left(\begin{array}{c}
-\operatorname{sgn}(n) h_{|n|-1, k}(\mathbf{r}) \\
h_{|n|, k}(\mathbf{r})
\end{array}\right)(11)
\end{aligned}
$$

for the other levels. In the Landau gauge $\mathbf{A}=B x \widehat{\mathbf{y}}$,

$$
\begin{aligned}
h_{n, k}(\mathbf{r})= & \left(\frac{1}{\pi \ell^{2} L_{y}^{2}}\right)^{1 / 4} \frac{1}{\sqrt{2^{n} n !}} e^{-i k y} \\
& \times H_{n}\left(\frac{x-k \ell}{\ell}\right) e^{-\frac{\left(x-k \ell^{2}\right)^{2}}{2 \ell^{2}}} .
\end{aligned}
$$

$$
\begin{aligned}
\Xi_{n, n^{\prime}}^{\sigma, \sigma}(\mathbf{q})= & \frac{1}{2} \Theta(|n|) \Theta\left(\left|n^{\prime}\right|\right)\left[F_{|n|,\left|n^{\prime}\right|}(\mathbf{q})+\operatorname{sgn}(n) \operatorname{sgn}\left(n^{\prime}\right) F_{|n|-1,\left|n^{\prime}\right|-1}(\mathbf{q})\right] \\
& +\frac{1}{\sqrt{2}}\left[\delta_{n, 0} \Theta\left(\left|n^{\prime}\right|\right)+\delta_{n^{\prime}, 0} \Theta(|n|)\right] F_{|n|,\left|n^{\prime}\right|}(\mathbf{q})+\delta_{n, 0} \delta_{n^{\prime}, 0} F_{0,0}(\mathbf{q})
\end{aligned}
$$

and

$$
\begin{aligned}
\Xi_{n, n^{\prime}}^{\sigma,-\sigma}(\mathbf{q})= & -\sigma \frac{1}{2} \Theta(|n|) \Theta\left(\left|n^{\prime}\right|\right)\left[\operatorname{sgn}(n) F_{|n|-1,\left|n^{\prime}\right|}(\mathbf{q}+\sigma 2 \mathbf{K})-\operatorname{sgn}\left(n^{\prime}\right) F_{|n|,\left|n^{\prime}\right|-1}(\mathbf{q}+\sigma 2 \mathbf{K})\right] \\
& +\sigma \frac{1}{\sqrt{2}}\left[\delta_{n, 0} \Theta\left(\left|n^{\prime}\right|\right) \operatorname{sgn}\left(n^{\prime}\right) F_{0,\left|n^{\prime}\right|-1}(\mathbf{q}+\sigma 2 \mathbf{K})-\delta_{n^{\prime}, 0} \Theta(|n|) \operatorname{sgn}(n) F_{|n|-1,0}(\mathbf{q}+\sigma 2 \mathbf{K})\right],
\end{aligned}
$$

where $\Theta(n)$ is the step function and

$$
F_{n, n^{\prime}\left(n \geq n^{\prime}\right)}(\mathbf{q})=\left(\frac{n^{\prime} !}{n !}\right)^{1 / 2}\left(\frac{\left(q_{y}+i q_{x}\right) \ell}{\sqrt{2}}\right)^{n-n^{\prime}} \exp \left(\frac{-q^{2} \ell^{2}}{4}\right) L_{n^{\prime}}^{n-n^{\prime}}\left(\frac{q^{2} \ell^{2}}{2}\right)
$$

with $F_{n, n^{\prime}\left(n \leq n^{\prime}\right)}(\mathbf{q})=\left[F_{n^{\prime}, n\left(n \geq n^{\prime}\right)}(-\mathbf{q})\right]^{*}$.

In our study of crystal states, we need matrix elements of the form $\Xi_{n, n^{\prime}}^{\sigma, \sigma^{\prime}}(\mathbf{G})$ with $G \sim 2 \pi n / a_{0}$ where $a_{0}$ is here the lattice constant of the Wigner or Skyrme crystals. We assume that the electronic density can be made small enough so that $a_{0}>>a$, the lattice constant of graphene. Moreover, although the summations over $\mathbf{G}$ extend to infinity in the formulas below, the exponential factor $\sim e^{-G^{2} \ell^{2} / 2}$ appearing in the functions $\Xi_{n, n^{\prime}}^{\sigma, \sigma^{\prime}}(\mathbf{G})$ makes these summations rapidly convergent if the filling factor is not too small. We thus have an effective cutoff value $G_{\max }$ such that $G_{\max }<<K$. It follows then that we can neglect the off diagonal matrix elements $\Xi_{n, n^{\prime}}^{\sigma, \sigma^{\prime} \neq \sigma}(\mathbf{G})$ that scatter electrons from one valley to an- 
other since they are very small in comparison with the other terms ${ }^{10}$. Essentially the same approximation was made in Ref. 6. We also make the usual approximation of neglecting Landau level mixing. This approximation is justified since the energy of the Landau levels are given by Eq. (17) so that the gap between the $n=0$ and $n=1$ Landau levels is thus $\Delta_{L . L .}=\sqrt{2} \hbar v_{F} / \ell \approx 424 \sqrt{B} \mathrm{~K}$ (with $B$ in Tesla) while the Coulomb interaction energy is of the order of $e^{2} / \kappa \ell=130 \sqrt{B} \mathrm{~K}$ (with $\kappa=5$ ). It was recently shown numerically that Landau level mixing is indeed negligible ${ }^{7,9}$.

With a Landé factor $g \approx 2$, the Zeeman gap $\Delta_{Z}=$ $g \mu_{B} B=1.34 B \mathrm{~K}$ is however quite small in comparison with the Coulomb energy, and the possibility of crystal states with spin as well as valley pseudospin textures can also be considered. Previous studies of analogous bilayer 2DEG systems ${ }^{14}$ suggest that groundstates with real spin admixed are rather fragile with respect to Zeeman coupling, and it seems unlikely that such a textured state would be stable for this value of $g$. Our preliminary studies of the phase diagram of the combined spin and valley pseudospin system confirm this conclusion 15 . In what follows we assume that the electronic spin is fully polarized so that we need only consider the valley degree of freedom.

In the Hartree-Fock approximation, our Hamiltonian becomes (apart from a constant term)

$$
\begin{aligned}
H= & N_{\phi} \sum_{\mathbf{q}} \sum_{\sigma, \sigma^{\prime}} H_{n}(\mathbf{q})\left\langle\rho_{n}^{\sigma, \sigma}(\mathbf{q})\right\rangle \rho_{n}^{\sigma^{\prime}, \sigma^{\prime}}(-\mathbf{q}) \\
& -N_{\phi} \sum_{\sigma, \sigma^{\prime}} \sum_{\mathbf{q}} X_{n}(\mathbf{q})\left\langle\rho_{n}^{\sigma, \sigma^{\prime}}(\mathbf{q})\right\rangle \rho_{n}^{\sigma^{\prime}, \sigma}(-\mathbf{q}),
\end{aligned}
$$

where $N_{\phi}$ is the Landau level degeneracy and we have defined the Hartree and Fock interactions

$$
\begin{aligned}
& H_{n}(\mathbf{q})=\left(\frac{e^{2}}{\kappa \ell}\right)\left(\frac{1}{q \ell}\right) \Xi_{n}(\mathbf{q}) \Xi_{n}(-\mathbf{q}), \\
& X_{n}(\mathbf{q})=\frac{1}{N_{\phi}}\left(\frac{e^{2}}{\kappa \ell}\right) \sum_{\mathbf{p}} H_{n}(\mathbf{p}) e^{i \mathbf{p} \times \mathbf{q} \ell^{2}},
\end{aligned}
$$

with the form factor

$$
\begin{aligned}
\Xi_{n}(\mathbf{q})= & \frac{1}{2} \Theta(|n|)\left[F_{|n|,|n|}(\mathbf{q})+F_{|n|-1,|n|-1}(\mathbf{q})\right] \\
& +\delta_{n, 0} F_{0,0}(\mathbf{q}) .
\end{aligned}
$$

In Eq. (18), we have defined the operators

$$
\rho_{n}^{\sigma, \sigma^{\prime}}(\mathbf{q}) \equiv \frac{1}{N_{\phi}} \sum_{k, k^{\prime}} e^{-\frac{i}{2} q_{x}\left(k+k^{\prime}\right) \ell^{2}} \delta_{k, k^{\prime}+q_{y}} c_{\sigma, n, k}^{\dagger} c_{\sigma^{\prime}, n, k^{\prime}}
$$

Note that, by definition,

$$
\left\langle\rho_{n}^{\sigma, \sigma}(\mathbf{q}=0)\right\rangle=\nu_{n, \sigma},
$$

where $\nu_{n, \sigma}=N_{n, \sigma} / N_{\phi}$ is the electronic filling factor of the $n^{\prime}$ th Landau level in the valley at $\sigma \mathbf{K}$. In a crystal, the average value $\left\langle\rho_{n}^{\sigma, \sigma^{\prime}}(\mathbf{q})\right\rangle$ is non zero only for $\mathbf{q}=\mathbf{G}$, a reciprocal lattice vector.

The ground-state energy per electron in Landau level $n$ is simply

$$
\begin{aligned}
\frac{E}{N_{n}}= & \frac{1}{2 \nu_{n}} \sum_{\mathbf{q}} \sum_{\sigma, \sigma^{\prime}} H_{n}(\mathbf{q})\left\langle\rho_{n}^{\sigma, \sigma}(\mathbf{q})\right\rangle\left\langle\rho_{n}^{\sigma^{\prime}, \sigma^{\prime}}(-\mathbf{q})\right\rangle \\
& -\frac{1}{2 \nu_{n}} \sum_{\sigma, \sigma^{\prime}} \sum_{\mathbf{q}} X_{n}(\mathbf{q})\left|\left\langle\rho_{n}^{\sigma, \sigma^{\prime}}(\mathbf{q})\right\rangle\right|^{2} .
\end{aligned}
$$

From Eq. (21), we see that the form factor $\Xi_{0}(\mathbf{q})$ for Landau level $n=0$ is exactly the same as for a $2 \mathrm{DEG}$ in a semiconductor quantum well or heterostructure. It follows that the phase diagram for graphene at low filling factor will be closely related to that of a conventional 2DEG with vanishing Zeeman gap ${ }^{16}$.

It is very useful to map the valley degree of freedom into a pseudospin $1 / 2$. Our convention is that a $K$ state is pseudospin up $(+)$ while $K^{\prime}$ is pseudospin down $(-)$. In this language, the components of the pseudospin vector $\operatorname{density} \mathbf{P}_{n}(\mathbf{q})=P_{n, x}(\mathbf{q}) \widehat{\mathbf{x}}+P_{n, y}(\mathbf{q}) \widehat{\mathbf{y}}+P_{n, z}(\mathbf{q}) \widehat{\mathbf{z}}$ are given by

$$
\begin{aligned}
& P_{n, x}(\mathbf{q})=\frac{\rho_{n}^{+,-}(\mathbf{q})+\rho_{n}^{-,+}(\mathbf{q})}{2}, \\
& P_{n, y}(\mathbf{q})=\frac{\rho_{n}^{+,-}(\mathbf{q})-\rho_{n}^{-,+}(\mathbf{q})}{2 i}, \\
& P_{n, z}(\mathbf{q})=\frac{\rho_{n}^{+,+}(\mathbf{q})-\rho_{n}^{-,-}(\mathbf{q})}{2},
\end{aligned}
$$

while the total density is given by

$$
\rho_{n}(\mathbf{q})=\rho_{n}^{+,+}(\mathbf{q})+\rho_{n}^{-,-}(\mathbf{q}) .
$$

In this language, Eq. (24) becomes

$$
\begin{aligned}
\frac{E}{N_{n}}= & \frac{1}{4 \nu_{n}}\left(\frac{e^{2}}{\kappa \ell}\right) \sum_{\mathbf{q}} \Upsilon_{n}(\mathbf{q})\left\langle\rho_{n}(-\mathbf{q})\right\rangle\left\langle\rho_{n}(\mathbf{q})\right\rangle \\
& +\frac{1}{\nu_{n}}\left(\frac{e^{2}}{\kappa \ell}\right) \sum_{\mathbf{q}} J_{n}(\mathbf{q})\left[\left\langle\mathbf{P}_{n}(-\mathbf{q})\right\rangle \cdot\left\langle\mathbf{P}_{n}(\mathbf{q})\right\rangle\right],
\end{aligned}
$$

with

$$
\begin{aligned}
& \Upsilon_{n}(\mathbf{q})=2 H_{n}(\mathbf{q})-X_{n}(\mathbf{q}) \\
& J_{n}(\mathbf{q})=-X_{n}(\mathbf{q})=-\int_{0}^{\infty} d y e^{-y^{2} / 2} J_{0}(y q \ell),
\end{aligned}
$$

where $J_{0}(x)$ is a Bessel function. For example, the liquid state at $\nu=1$ has an energy given by

$$
\frac{E}{N_{n}}=-\left[\frac{1}{4} X_{n}(0)+X_{n}(0)\left|\left\langle\mathbf{P}_{n}(0)\right\rangle\right|^{2}\right],
$$

and $\left|\left\langle\mathbf{P}_{n}(0)\right\rangle\right|=1 / 2$. (We have taken into account the positive background to cancel the divergence of $\left.H_{n}(0)\right)$. It follows that this liquid state is fully pseudospin polarized but the direction of polarization is arbitrary. This is 
also true for the crystal states (see below) i.e. our hamiltonian has an $S U(2)$ symmetry. We deduce that both states support a pseudospin wave Goldstone mode with a $q^{2}$ dispersion at long wavelength.

We remark that, in view of Eqs. (8)-(9), the pseudospin degree of freedom is equivalent to the sublattice degree of freedom for Landau level $n=0$. This is not true, however, for other values of $n$.

\section{SINGLE AND TWO-PARTICLE GREEN'S FUNCTIONS}

The average values $\left\langle\rho_{n}^{\sigma, \sigma^{\prime}}(\mathbf{B})\right\rangle$ describing the crystal states can be extracted from the Matsubara singleparticle Green's function $G_{n}$ which is defined by (with $\left.X=k \ell^{2}\right)$

$$
G_{n}^{\sigma, \sigma^{\prime}}\left(X, X^{\prime}, \tau\right)=-\left\langle T c_{\sigma, n, X}(\tau) c_{\sigma^{\prime}, n^{\prime}, X^{\prime}}^{\dagger}(0)\right\rangle .
$$

Its Fourier transform is

$$
\begin{aligned}
& G_{n}^{\sigma, \sigma^{\prime}}\left(\mathbf{G}, \tau=0^{-}\right)=\frac{1}{N_{\phi}} \sum_{X, X^{\prime}} e^{-\frac{i}{2} G_{x}\left(X+X^{\prime}\right)} \\
& \times \delta_{X, X^{\prime}-G_{y} l_{\perp}^{2}} G_{n}^{\sigma, \sigma^{\prime}}\left(X, X^{\prime}, \tau\right),
\end{aligned}
$$

so that

$$
\left\langle\rho_{n}^{\sigma^{\prime}, \sigma}(\mathbf{G})\right\rangle=G_{n}^{\sigma, \sigma^{\prime}}\left(\mathbf{G}, \tau=0^{-}\right)
$$

The equation of motion for the Green's function in the Matsubara formalism is obtained by using the Heisenberg equation

$$
\hbar \frac{\partial}{\partial \tau}(\ldots)=\left[H_{H F}-\mu N,(\ldots)\right]
$$

and is given by

$$
\begin{aligned}
& \hbar\left(i \omega_{n}-\mu\right) G_{n}^{\sigma, \sigma^{\prime}}\left(\mathbf{G}, \omega_{n}\right) \\
& -\sum_{\sigma^{\prime \prime}} \sum_{\mathbf{G}^{\prime}} F_{\mathbf{G}, \mathbf{G}^{\prime}}^{\sigma, \sigma^{\prime \prime}} e^{-i \mathbf{G} \times \mathbf{G}^{\prime} \ell^{2} / 2} G_{n}^{\sigma^{\prime \prime}, \sigma^{\prime}}\left(\mathbf{G}^{\prime}, \omega_{n}\right) \\
= & \hbar \delta_{\mathbf{G}, 0} \delta_{\sigma, \sigma^{\prime}}
\end{aligned}
$$

where $\omega_{n}$ is a fermionic Matsubara frequency and the matrix elements $F_{\mathbf{G}, \mathbf{G}^{\prime}}^{\sigma, \sigma^{\prime}}$ are given by

$$
\begin{aligned}
F_{n}^{\sigma, \sigma^{\prime}}\left(\mathbf{G}, \mathbf{G}^{\prime}\right)= & H_{n}\left(\mathbf{G}-\mathbf{G}^{\prime}\right) \sum_{\sigma^{\prime}}\left\langle\rho_{n}^{\sigma^{\prime}, \sigma^{\prime}}\left(\mathbf{G}-\mathbf{G}^{\prime}\right)\right\rangle \delta_{\sigma, \sigma^{\prime}} \\
& -X_{n}\left(\mathbf{G}-\mathbf{G}^{\prime}\right)\left\langle\rho_{n}^{\sigma^{\prime}, \sigma}\left(\mathbf{G}-\mathbf{G}^{\prime}\right)\right\rangle
\end{aligned}
$$

To find the order parameters $\left\langle\rho_{n}^{\sigma^{\prime}, \sigma}(\mathbf{G})\right\rangle$, we solve the Hartree-Fock equation of motion numerically by an iterative method. The procedure is described in detail in Ref. 17.

In order to compute the collective excitations, we define the 16 response functions

$$
\begin{aligned}
\chi_{n}^{a, b, c, d}\left(\mathbf{q}, \mathbf{q}^{\prime} ; \tau\right)= & -N_{\phi}\left\langle T \rho_{n}^{a, b}(\mathbf{q}, \tau) \rho_{n}^{c, d}\left(-\mathbf{q}^{\prime}, 0\right)\right\rangle \\
& +N_{\phi}\left\langle\rho_{n}^{a, b}(\mathbf{q})\right\rangle\left\langle\rho_{n}^{c, d}\left(-\mathbf{q}^{\prime}\right)\right\rangle,
\end{aligned}
$$

where $a, b, c, d$ are valley indices. For a crystal, $\mathbf{q}=\mathbf{k}+\mathbf{G}$ and $\mathbf{q}=\mathbf{k}+\mathbf{G}^{\prime}$, where $\mathbf{k}$ is a vector in the first Brillouin zone. In the generalized randomphase approximation (GRPA), the equation of motion for $\chi_{n}^{a, b, c, d}\left(\mathbf{q}, \mathbf{q}^{\prime} ; i \Omega_{n}\right)$ is given by

$$
\sum_{\mathbf{q}^{\prime \prime}}\left[i \Omega_{n} I-\frac{1}{\hbar} F\left(\mathbf{q}, \mathbf{q}^{\prime \prime}\right)\right] \chi\left(\mathbf{q}^{\prime \prime}, \mathbf{q}^{\prime} ; i \Omega_{n}\right)=D\left(\mathbf{q}, \mathbf{q}^{\prime}\right)
$$

where $I$ is the $4 N_{\mathbf{q}} \times N_{\mathbf{q}}$ unit matrix with $N_{\mathbf{q}}$ the number of vectors $\mathbf{G}$ kept in the calculation and we have defined the matrices:

$$
\chi=\left(\begin{array}{cccc}
\chi_{+,+,+,+} & \chi_{+,+,-,+} & \chi_{+,+,+,-} & \chi_{+,+,-,-} \\
\chi_{+,-,+,+} & \chi_{+,-,-,+} & \chi_{+,-,+,-} & \chi_{+,-,-,-} \\
\chi_{-,+,+,+} & \chi_{-,+,-,+} & \chi_{-,+,+,-} & \chi_{-,+,-,-} \\
\chi_{-,-,+,+} & \chi_{-,-,-,+} & \chi_{-,-,+,-} & \chi_{-,-,-,-}
\end{array}\right)
$$

and

$$
\begin{aligned}
F\left(\mathbf{q}, \mathbf{q}^{\prime}\right)= & U\left(\mathbf{q}, \mathbf{q}^{\prime}\right) \\
& -\sum_{\mathbf{q}^{\prime \prime}} D\left(\mathbf{q}, \mathbf{q}^{\prime \prime}\right)\left(\widetilde{H}\left(\mathbf{q}^{\prime \prime}, \mathbf{q}^{\prime}\right)-\widetilde{X}\left(\mathbf{q}^{\prime \prime}, \mathbf{q}^{\prime}\right)(\dot{\gamma})\right)
\end{aligned}
$$

with

$$
D=\left(\begin{array}{cccc}
2 i \alpha\left\langle\rho_{n}^{+,+}\right\rangle & -\left\langle\rho_{n}^{-,+}\right\rangle \gamma & \left\langle\rho_{n}^{+,-}\right\rangle \gamma^{*} & 0 \\
-\left\langle\rho_{n}^{+,-}\right\rangle \gamma & \left\langle\rho_{n}^{+,+}\right\rangle \gamma^{*}-\left\langle\rho_{n}^{-,-}\right\rangle \gamma & 0 & \left\langle\rho_{n}^{+,-}\right\rangle \gamma^{*} \\
\left\langle\rho_{n}^{-.+}\right\rangle \gamma^{*} & 0 & \left\langle\rho_{n}^{-,-}\right\rangle \gamma^{*}-\left\langle\rho_{n}^{+,+}\right\rangle \gamma & -\left\langle\rho_{n}^{-,+}\right\rangle \gamma \\
0 & \left\langle\rho_{n}^{-,+}\right\rangle \gamma^{*} & -\left\langle\rho_{n}^{+,-}\right\rangle \gamma & 2 i \alpha\left\langle\rho_{n}^{-,},-\right\rangle
\end{array}\right)
$$


and

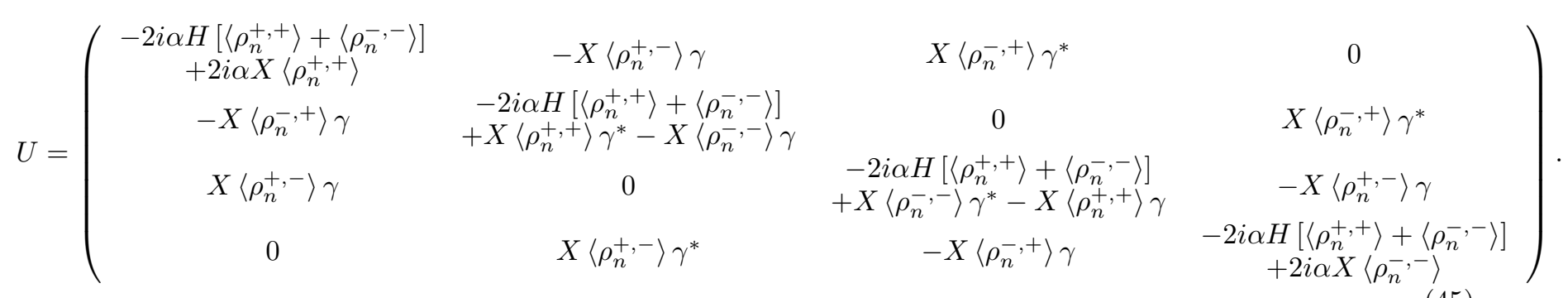

In these equations, we adopt the conventions that $\left\langle\rho^{a, b}\right\rangle=\left\langle\rho^{a, b}\left(\mathbf{q}-\mathbf{q}^{\prime}\right)\right\rangle, H\left\langle\rho^{a, b}\right\rangle$ stands for $H_{n}\left(\mathbf{q}-\mathbf{q}^{\prime}\right)\left\langle\rho^{a, b}\left(\mathbf{q}-\mathbf{q}^{\prime}\right)\right\rangle, \gamma=e^{-i \mathbf{q} \times \mathbf{q}^{\prime} \ell^{2} / 2}$, and $\alpha=$ $\sin \left(\frac{\mathbf{q} \times \mathbf{q}^{\prime} \ell^{2}}{2}\right)$. The Hartree and Fock interaction matrices are given by

$$
\widetilde{H}=\left(\begin{array}{cccc}
H_{n}(\mathbf{q}) & 0 & 0 & H_{n}(\mathbf{q}) \\
0 & 0 & 0 & 0 \\
0 & 0 & 0 & 0 \\
H_{n}(\mathbf{q}) & 0 & 0 & H_{n}(\mathbf{q})
\end{array}\right) \delta_{\mathbf{q}, \mathbf{q}^{\prime}}
$$

and

$$
\tilde{X}=\left(\begin{array}{cccc}
X_{n}(\mathbf{q}) & 0 & 0 & 0 \\
0 & X_{n}(\mathbf{q}) & 0 & 0 \\
0 & 0 & X_{n}(\mathbf{q}) & 0 \\
0 & 0 & 0 & X_{n}(\mathbf{q})
\end{array}\right) \delta_{\mathbf{q}, \mathbf{q}^{\prime}}
$$

Once the Hartree-Fock densities $\left\langle\rho^{a, b}\right\rangle$ are calculated for the crystal state considered, the response functions can be computed using Eq. (40). The collective excitations appear as poles of these response functions. To derive the dispersion relations, we follow the poles in the response functions as the wave vector $\mathbf{k}$ is varied within the first Brillouin zone. We consider only the low-energy modes in the present work. The response functions also have higher-energy modes corresponding to more localized excitations.

The various response functions in Eq. (41) can be combined in an obvious way to give the pseudospin response functions $\chi_{\rho_{n}, \rho_{n}}, \chi_{S_{x}, S_{x}}, \chi_{S_{y}, S_{y}}$, and $\chi_{S_{z}, S_{z}}$.

\section{PHASE DIAGRAM AND COLLECTIVE MODES}

In the absence of Zeeman coupling, each Landau level has a fourfold degeneracy (the valley degeneracy combined with the usual spin doublet). For undoped graphene, the $n=0$ Landau level multiplet is half-filled. We use the notation $\nu_{n} \in[0,4]$ for the filling factor of each Landau level multiplet. The total filling factor is thus given by $\nu=4 n-2+\nu_{n}$. In this work, we assume a finite Zeeman coupling but neglect any mixing of Landau level with different spins so that the phase diagram for $\nu_{n} \in[0,2]$ is identical with that for $\nu_{n} \in[2,4]$. Without lost of generality, we consider $\nu_{n} \in[0,2]$ from now on.

Our procedure for solving Eq. (37) does not allow us to find the absolute ground state of the 2DEG for a given filling factor. Instead, we have to be content with comparing the energy of different phases and finding the lowest one amongst them. In this study, we focus on the crystal states and more specifically on the Skyrme crystals. The filling factor $\nu_{n}$ is that of the partially filled Landau level and all filled levels below $n$ are assumed inert. This procedure is valid when Landau level mixing is small, provided one considers only intra-Landau level excitations $\underline{9.18}$. We consider the following states in each Landau level $n$ :

1. Electron bubble crystal (eBCn). A triangular lattice with $N_{c}$ electrons per unit cell and filling factor $\nu_{n}<0.5$. More precisely, bubbles are maximal density droplets 4.5 .

2. Hole bubble crystal $(\mathrm{hBCn})$. A triangular lattice with $N_{c}$ holes per unit cell and filling factor $0.5<$ $\nu_{n}<1$. The lattice constant $a_{0}$ of such a crystal is determined by the relation $2 \pi n_{h} \ell^{2}=\left|\nu_{n}-1\right|$ where $n_{h}=N_{c} / \varepsilon a_{0}^{2}$ is the hole density with $N_{c}$ the number of holes in the bubbles and $\varepsilon=\sqrt{3} / 2$ for a triangular lattice. Note that we find both the $\mathrm{hBCn}$ state and the meron crystal considered below to be lower in energy that the eBCn groundstate assumed in Ref. 6 in the same range of filling factors.

3. Meron crystal (MC). A square lattice with four merons of charge $-e / 2\left(\right.$ if $\left.\nu_{n}<1\right)$ or $e / 2$ (if $\nu_{n}>1$ ) per unit cell, equally spaced and assembled in a checkerboard configuration. The $z$ component of the pseudospin and the vorticities alternate from one site to the next and two of the merons in the unit cell have a global pseudospin phase in the $x-y$ plane which is opposite to the two others. In semiconductor 2DEG's, this configuration is found for the (spin) skyrmion crystal when the Zeeman energy is zero 12 . This crystal is represented in Fig. 1.

4. Meron pair crystal (MPC). A triangular lattice with four merons per unit cell. At each lattice site, two merons with the same value of $P_{z}$ and 
vorticities but opposite values of the global phase are coupled together so that the pseudospins rotate by $4 \pi$ on a path encircling the two meron pairs. This configuration is represented in Fig. 2, The merons are not equally spaced. The possibility for skyrmions of opposite phases to form pairs was considered in Ref. 19. The MC and MPC phases are in competition with each other and their energies are very close. We remark that, in the absence of an equivalent Zeeman coupling, $\Delta_{Z}$, a single skyrmion should have a size comparable to that of the sample size. In a lattice, this causes a strong interaction between skyrmions that leads to a lattice of merons even when the skyrmion filling factor $\left|\nu_{n}-1\right| \rightarrow 0$. It is important to notice that the limits $\Delta_{Z} \rightarrow 0$ and $\left|\nu_{n}-1\right| \rightarrow 0$ do not commute $\frac{12}{2}$. If we were to choose $\left|\nu_{n}-1\right| \rightarrow 0$ first and then $\Delta_{Z} \rightarrow 0$, we would find instead a Skyrme crystal ${ }^{12}$.

For the numerical calculations, we consider a filling factor $\nu_{n} \in[0.1,0.9]$. For $\nu_{n}<0.1$ or $\left|\nu_{n}-1\right|<0.1$, the number of reciprocal lattice vectors needed in the calculation becomes very large and we do not get good convergence. This is due to the fact that the size in real space of the quasiparticles (electrons for $\nu_{n}<0.1$ or holes or skyrmions for $\left.\left|\nu_{n}-1\right|<0.1\right)$ decreases so that more wavevectors are needed to describe them. Also, the hamiltonian has electron-hole symmetry around $\nu_{n}=1$ so that the sequence of phase transitions found for $\nu_{n}>1$ is the mirror image of that for $\nu_{n}<1$ with particles replaced by anti-particles. For example, the couterpart of the phase $\mathrm{eBC} 1$ at $\nu_{n}=0.2$ is a $\mathrm{hBC} 1$ at $\nu_{n}=1.8$ with a filling of holes given by $\nu_{n, h}=2.0-1.8=0.2$. Similarly, the counterpart of a crystal of merons (with charge $-e / 2$ ) at $\nu_{n}=0.8$ (with a filling of merons given by $\left.\nu_{n, m}=1.0-0.8=0.2\right)$ is a crystal of anti-merons (charge $+e / 2$ ) at $\nu_{n}=1.2$ with a filling of anti-merons given by $\nu_{n, a m}=1.2-1.0=0.2$.

We show in Fig. 3 the energies of different phases of the 2DEG in graphene for Landau level $n=0$. We find the following sequence: $\mathrm{eBC} 1$ for $\nu_{0} \in[0.1,0.5], \mathrm{hBC} 1$ for $\nu_{0} \in[0.5,0.55], \mathrm{MC}$ for $\nu_{0} \in[0.55,0.65]$, and $\mathrm{MCP}$ for $\nu_{0} \in[0.65,0.9]$. As noted above, this sequence of transitions is the same as that calculated for a 2DEG in GaAs-AlGaAs quantum wells in the absence of Zeeman coupling because the effective interactions $H_{0}(\mathbf{q})$ and $X_{0}(\mathbf{q})$ are the same in both cases. To determine this sequence, we not only find the state with the lowest energy but also compute the collective mode spectrum in order to check that the crystal is stable. For the MC and MPC where the difference in energy is close to our numerical accuracy, the stability criteria allows us to find the correct ground state.

The eBC1 phase is fully pseudospin polarized but its energy is independent of the orientation of the pseudospins. The crystal thus has a full SU(2) symmetry for the pseudospin. The dispersion relations of the two Goldstone modes of this crystal is given in Fig. 4. The dispersion is plotted along the path

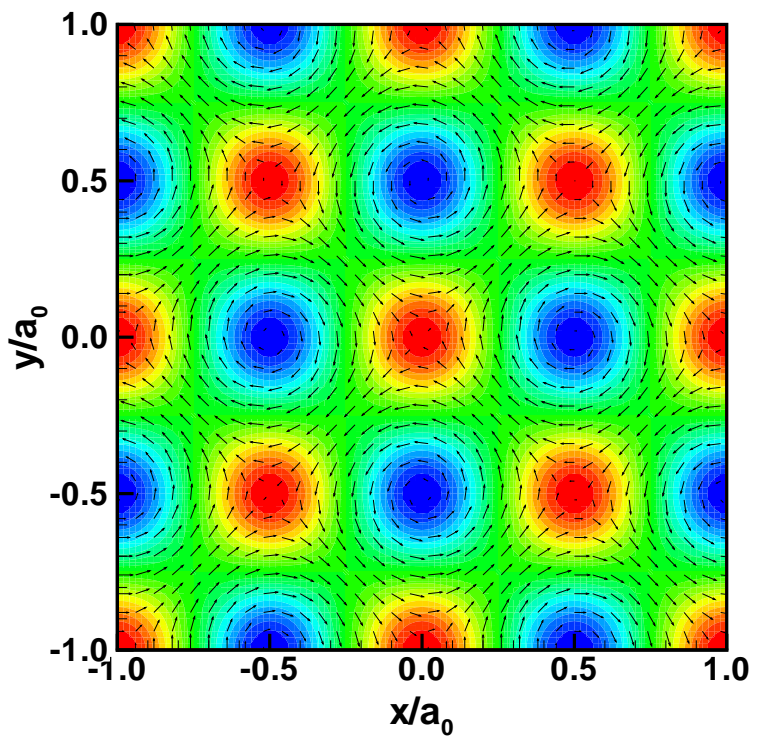

FIG. 1: Pseudospin texture in a meron crystal at filling factor $\nu_{0}=0.8$ in Landau level $n=0$. The crystal has four merons per unit cell. In each unit cell, two merons with the same vorticity have opposite phases as explained in the text. Contours (ranging from -0.5 to 0.5 ) indicate the $z$ component of the pseudospin with dark regions corresponding to positive values.

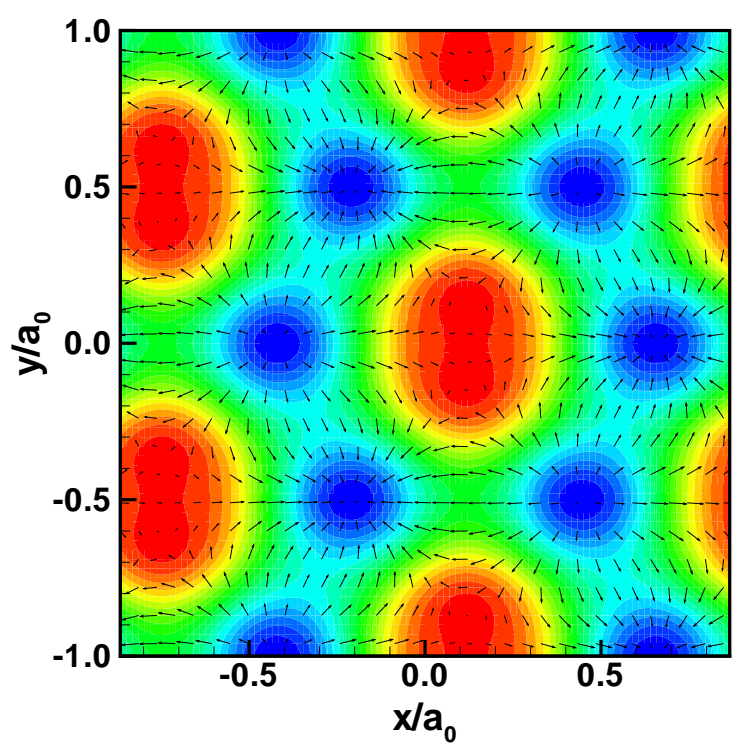

FIG. 2: Pseudospin texture in a meron pair crystal at filling factor $\nu_{0}=0.8$ in Landau level $n=0$. The lattice is triangular and there are four merons per unit cell. Merons are bound in pairs with same value of $P_{z}$ and vorticities but opposite phases at each lattice site. Contours (ranging from -0.5 to 0.5 ) indicate the $z$ component of the pseudospin with dark regions corresponding to positive values. 


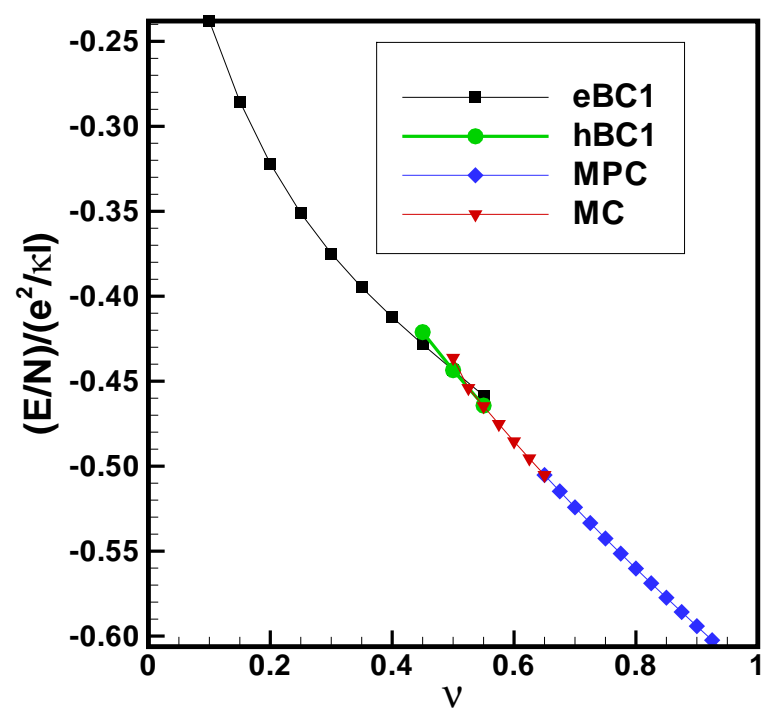

FIG. 3: Hartree-Fock energy per electron as a function of filling factor for various crystal phases in Landau level $n=0$.

$\Gamma-J-X-\Gamma$,corresponding to the wave vectors $\left(k_{x}, k_{y}\right)=$ $(0,0),\left(2 \pi_{0} / a\right)(1 / \sqrt{3}, 1 / 3),\left(2 \pi / a_{0}\right)(1 / \sqrt{3}, 0),(0,0)$.

The wave vector $k$ represents the total distance, in reciprocal space and in units of $2 \pi / a_{0}$ along the path $\Gamma-J-X-\Gamma$ from the origin $\Gamma$. The legend indicates in what response function $\chi_{\rho_{n}, \rho_{n}}, \chi_{s_{x}, s_{x}}, \chi_{s_{y}, s_{y}}$, or $\chi_{s_{z}, s_{z}}$ the collective mode has the biggest weight. This gives an indication of the nature of the mode. In Fig. 4 the phonon mode has its biggest weight in $\chi_{\rho_{n}, \rho_{n}}$ and $\chi_{S_{x}, S_{x}}$ while the pseudospin wave mode has its weight in $\chi_{S_{y}, S_{y}}$ and $\chi_{S_{z}, S_{z}}$. That, is, since we forced the pseudospin to be polarized along the $x$ direction, the pseudospin wave mode corresponds to a precession of the pseudospin about the $x$ axis. The phonon dispersion is typical of what is found for a Wigner crystal17. It is gapless, with $\omega \sim q^{3 / 2}$ behavior at small wave vector. For the pseudospin wave, the dispersion is $\omega \sim q^{2}$ at small wave vector confirming the $\mathrm{SU}(2)$ symmetry. At $\nu_{0}=0.2$, the bandwidth of the pseudospin mode is two orders of magnitude smaller than that of the phonon mode. While the bandwidth of the phonon mode does not change much as $\nu_{0}$ increases to $\nu_{0}=0.5$, that of the pseudospin mode changes dramatically, becoming of the same order as that of the phonon mode at $\nu_{0}=0.5$. The pseudospin stiffness thus increases rapidly with $\nu_{0}$. The $\mathrm{hBC} 1$ dispersion has the same features as the $\mathrm{eBC} 1$ as can be seen in Fig. 5 .

For $\nu>0.55$ in $n=0$, we find that, within our numerical accuracy, the MC and MPC have the same energy and are lower in energy than the other phases considered. The dispersion relations, however, indicate that the $\mathrm{MC}$ is stable in the range $\nu \in[0.55,0.65]$

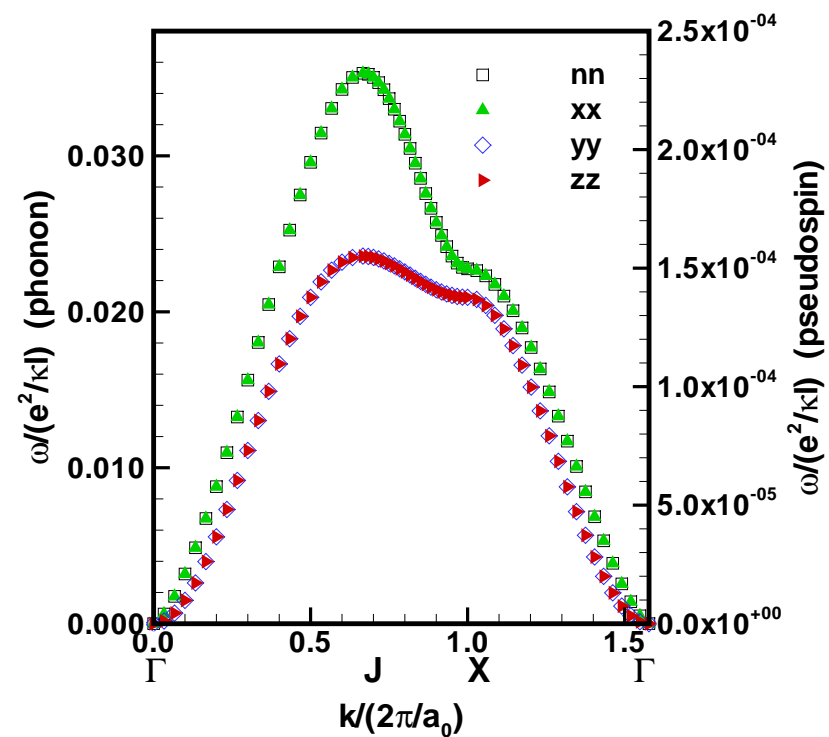

FIG. 4: Dispersion relation of the two Goldstone modes of the eBC1 state at $\nu_{0}=0.2$ in Landau level $n=0$. The dispersion is plotted along the irreducible Brillouin zone of the triangular lattice. The left (right) $y$ axis gives the phonon (pseudospin) frequency. The phonon mode has its biggest weight in $\chi_{\rho_{n}, \rho_{n}}$ and $\chi_{S_{x}, S_{x}}$ while the pseudospin wave mode has its biggest weight in $\chi_{S_{y}, S_{y}}$ and $\chi_{S_{z}, S_{z}}$

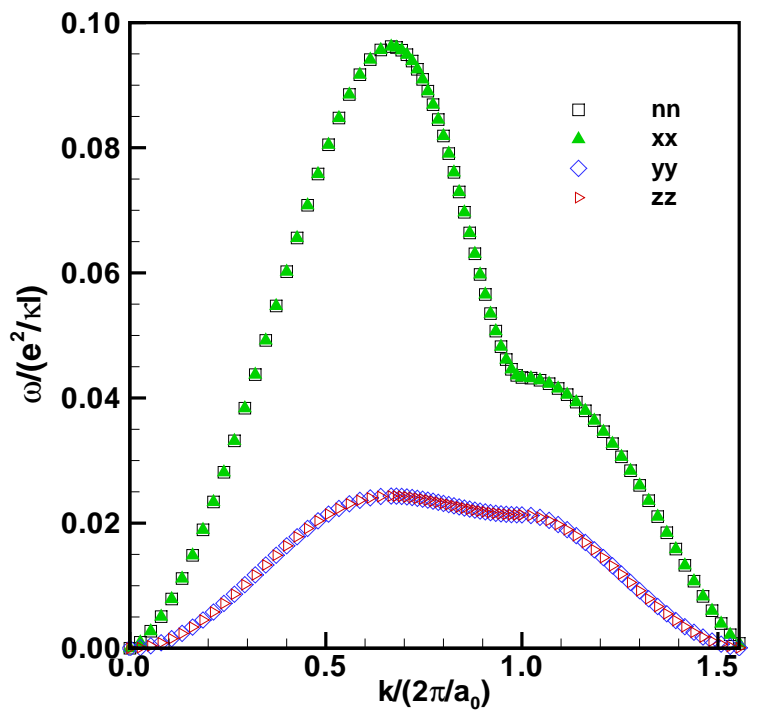

FIG. 5: Dispersion relation of the two Goldstone modes of the $\mathrm{hBC} 1$ state at $\nu_{1}=0.55$ in Landau level $n=1$. The dispersion is plotted along the irreducible Brillouin zone of the triangular lattice. The phonon mode has its biggest weight in $\chi_{\rho_{n}, \rho_{n}}$ and $\chi_{S_{x}, S_{x}}$ while the pseudospin wave mode has its biggest weight in $\chi_{S_{y}, S_{y}}$ and $\chi_{S_{z}, S_{z}}$ 
while the MPC is unstable in that range and vice versa for $\nu \in[0.65,0.90]$ so that there is a phase transition between these two states. We show in Figs. 6] and 7 the dispersion relations for these two states. For the MC phase, the dispersion is plotted along the path $\Gamma-$ $M-X-\Gamma$, corresponding to the wave vectors $\left(k_{x}, k_{y}\right)=$ $(0,0),\left(2 \pi_{0} / a\right)(1 / 2,1 / 2),\left(2 \pi / a_{0}\right)(1 / 2,0),(0,0) \quad$ since the unit cell is that of a square lattice. The dispersion in both cases show the usual gapless phonon mode with $\omega \sim q^{3 / 2}$ behavior at small wave vector which appears as a pole of $\chi_{\rho_{n}, \rho_{n}}$ and 3 other linearly dispersing Goldstone modes. Some of the pseudospin modes are degenerate along sections of the contour of the irreducible Brillouin zone. The degeneracy of some of these modes is only lifted along $\Gamma-X$ in the MC phase in 7 . For wave vector $\mathbf{k}$ in an arbitrary direction, however, the 3 pseudospin modes are non degenerate.

The energy of the two meron lattices are invariant under a rotation of the pseudospin texture around the $x, y$ or $z$-axis since there is no equivalent of the Zeeman coupling in the graphene 2DEG. This implies there are three independent ways to rotate the spins of the state without any cost in energy, leading to the three Goldstone modes found in the GRPA. Animations of these three modes support this interpretation ${ }^{16}$.

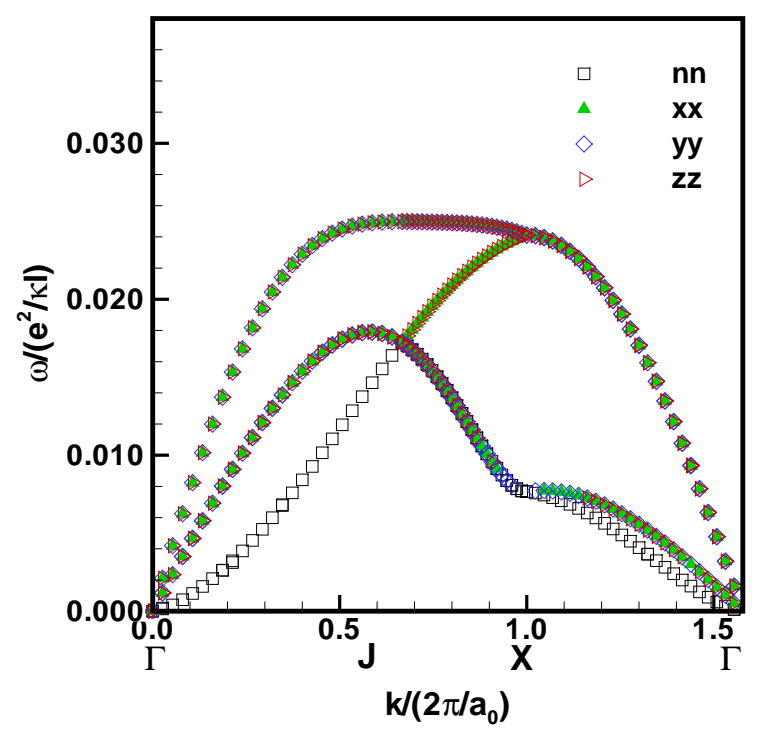

FIG. 6: Dispersion relation of the Goldstone modes of the MPC state at $\nu_{0}=0.8$ in Landau level $n=0$. The dispersion is plotted along the irreducible Brillouin zone of the triangular lattice. The legend indicates in what response function each collective mode has its biggest weight.

We show in Fig. 8 the energies of different phases of the 2DEG in graphene for Landau level $n=1$. We find the following sequence: eBC1 for $\nu_{1} \in[0.1,0.5]$, hBC1 for $\nu_{1} \in[0.5,0.75], \mathrm{MC}$ for $\nu_{1} \in[0.75,0.80]$ and MPC for $\nu_{1} \in[0.75,0.90]$. The MC and MPC phases have almost

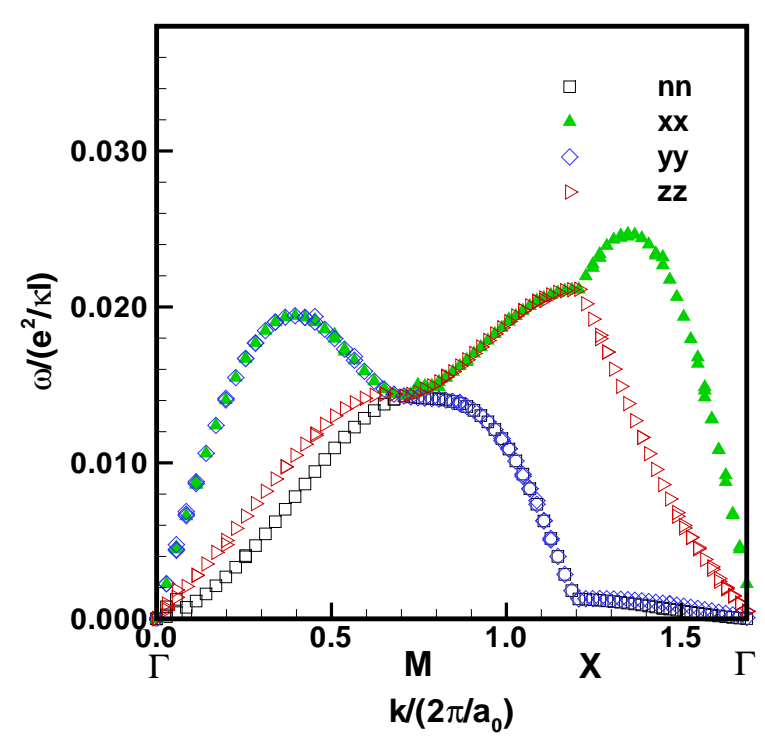

FIG. 7: Dispersion relation of the Goldstone modes of the MC state at $\nu_{0}=0.625$ in Landau level $n=0$. The dispersion is plotted along the irreducible Brillouin zone of the square lattice. The legend indicates in what response function each collective mode has its biggest weight.

the same energy within our numerical accuracy so that these two phases are represented by the line MC-MPC in 8. In comparison with the case $n=0$, we see that the filling factor range for which a pseudospin texture exists for $n=1$ has decreased relative to $n=0$. In contrast with what happens in a conventional 2DEG, however, there is a possibility for such textures in Landau level $n=$ 1. This is due to the fact that the effective interactions in the two systems are now different in view of Eq. (21).

The dispersion of the gapless modes in Landau levels $n=1$ and $n=2$ are similar to what is seen in $n=0$ as can be seen from Fig. 5. Bubble crystals with more than one electron per site have additional gapped modes related to internal excitations of the bubbles; we do not focus on these modes in this work ${ }^{5}$.

Fig. 9 shows the phase diagram of the 2DEG in graphene for Landau level $n=2$. We find here a sequence of transitions involving electron and hole bubble crystals with one or two electrons per bubble. The two meron phases MC and MPC have higher energy than the other phases considered so that there are no meron crystals of these types in $n=2$ and most probably in higher Landau levels as well. We emphasized however that our calculation is restricted to the range of partial filling factor $\nu-1>0.1$. Because the pseudospin textured states are pushed closer to $\nu=1$ as $n$ increases, we cannot rule the existence of meron crystals for $n \geq 2$. Indeed, Yang, Das Sarma and MacDonald have shown that skyrmions are the lowest-energy charged excitations in graphene for Landau levels up to $n=3^{11}$. Meron crystals could thus 


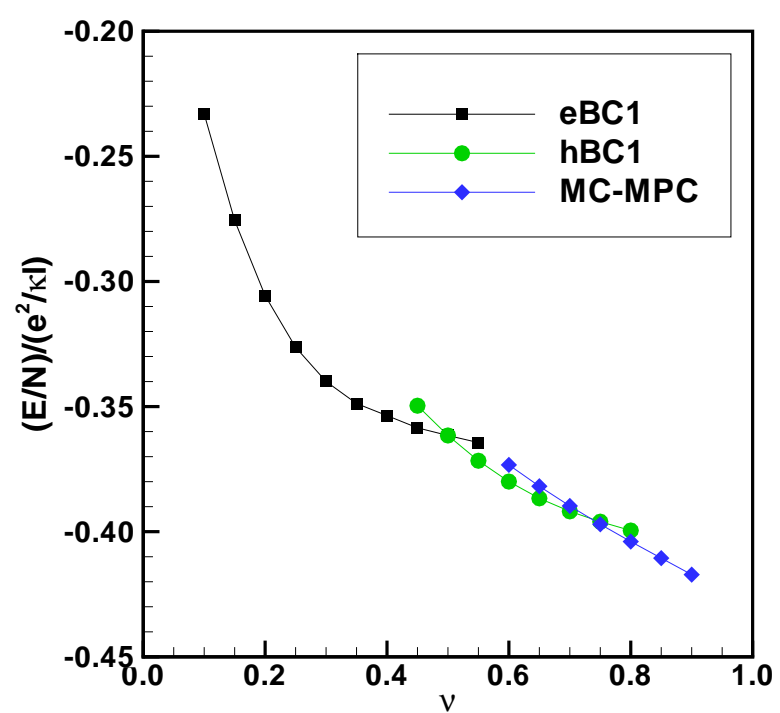

FIG. 8: Hartree-Fock energy per electron as a function of filling factor for various crystal phases in Landau level $n=1$.

also be present in Landau levels $n=2,3$.

For $n=2$ and near filling factor $\nu_{2}=0.5$, none of the phases that we considered in our analysis are stable so that the ground state must be of another crystal type, most probably the stripe phase if we compare with the situation in semiconductor 2DEG. This is also the conclusion of Hartree-Fock calculations in Refs. 6 and 9 . A stripe state probably occurs near $\nu_{n}=0.5$ for Landau level $n \geq 2$ in the HFA.

\section{SIGNATURES OF WIGNER AND MERON CRYSTALS IN PINNING BEHAVIOR}

Generally, collective modes of 2DEG's are detected by inelastic light scattering or via microwave absorption. The latter experiments are most sensitive to the long wavelength, low frequency behavior of the collective modes. A disorder potential pins the Wigner crystal, in the sense that the phonon mode becomes gapped at a pinning frequency $\omega_{p}$ that is dependent on the strength of the potential and on that of the magnetic field. The behavior of the pinning frequency with magnetic field depends critically on the interplay between different length scales: the size of the electron wavefunction on each lattice site, the lattice periodicity, and the magnetic length ${ }^{20,21}$. The pinning frequency in the longitudinal conductivity may be inferred from results such as those found here using the replica trick ${ }^{22}$.

In the graphene case, both the pseudospin and phonon mode involve charge fluctuations so we may speculate that the pseudospin mode will also be pinned in the presence of disorder, in the sense of opening a gap in their

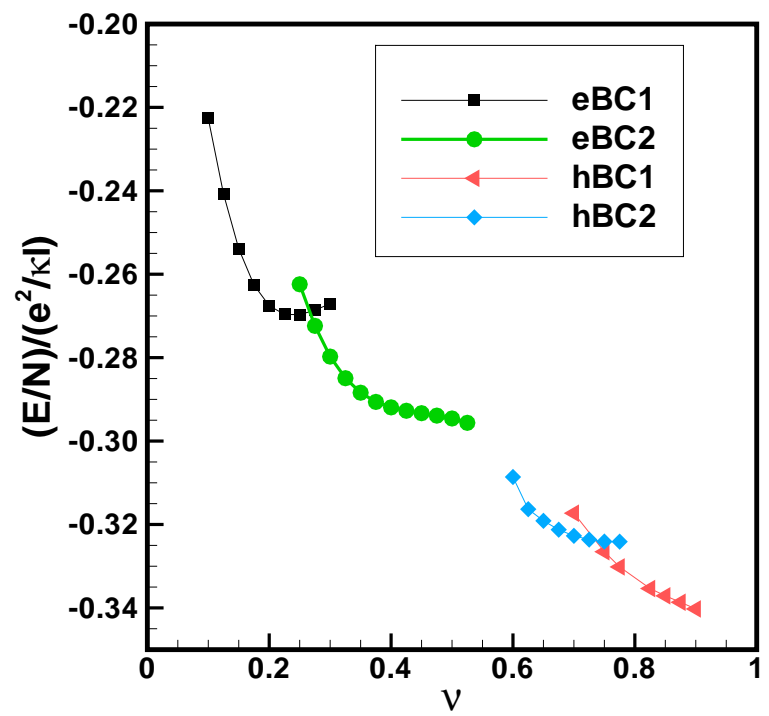

FIG. 9: Hartree-Fock energy per electron as a function of filling factor for various crystal phases in Landau level $n=2$.

spectrum, because the latter generically breaks pseudospin symmetry. Disorder should also pin the four Goldstone modes of the meron crystals: the phonon mode and the three pseudospin wave modes. If such pinned modes are separately observable, they could provide a unique signature of the formation of a Wigner or meron crystals in graphene. This conclusion should be contrasted with the case of a semiconductor 2DEG. There, a Wigner crystal has only one gapless (phonon) mode at finite Zeeman coupling. A skyrmion (finite Zeeman coupling) or meron (zero Zeeman coupling) crystal has one phonon mode which is gapped and one (Wigner) or three (meron) gapless spin wave modes that remain gapless in the presence of disorder.

For the pinning modes to be visible in microwave absorption experiments ${ }^{3}$, they must show up in $\Re\left[\sigma_{x x}(\mathbf{k} \rightarrow 0, \omega)\right]$ where $\sigma$ is the conductivity tensor. Equivalently, they must appear as poles of the currentcurrent response functions $\Im\left[\chi_{i, i}^{J, J}(\mathbf{k} \rightarrow 0, \omega)\right]$ with $i=$ $x, y$. A calculation of the conductivity tensor in the presence of disorder is difficult for the crystal states. For a simple Wigner crystal (one phonon mode only), it can be done by mapping the system to an effective harmonic model ${ }^{20,22}$ and using the replica trick. So far there has been no generalization of this method to crystals with an additional layer or valley degree of freedom. To test our speculation that the phonon and valley pseudospin modes are visible in microwave absorption, we "simulate" a disorder potential by adding a periodic external potential. Note that our HFA method forces us to choose this periodicity to be the same as that of the crystal considered. 
In formulating the relevant response function for conductivity, the current operator is built up from operators that excite electrons between Landau levels. This presents problems when the Hilbert space is restricted to one Landau level as in our calculation. In principle one needs to retain other Landau levels in order to obtain a non-vanishing result, significantly complicating the calculations. To circumvent this, we wish to find an appropriate projection of the current operator into a single Landau level. To do this, we generalize a method first introduced by Girvin, MacDonald and Platzman in Ref. 23. This procedures captures the drift current $\mathbf{j}(\mathbf{r})=\frac{c e}{B} \rho(\mathbf{r}) \nabla U(\mathbf{r}) \times \widehat{\mathbf{z}}$ of the electrons in the potential $U(\mathbf{r})$ and gives a current that satisfies the continuity equation. Improvements upon this procedure are possible but lead to very complicated expressions ${ }^{24}$. We first write a second quantized hamiltonian including the (total) density $\rho(\mathbf{k})$ and valley-pseudospin operator $\mathbf{P}(\mathbf{k})$

$$
\begin{aligned}
H= & N_{\varphi} \frac{1}{4}\left(\frac{e^{2}}{\kappa \ell}\right) \sum_{\mathbf{q}} \Upsilon(\mathbf{q}) \rho(-\mathbf{q}) \rho(\mathbf{q}) \\
& -N_{\varphi}\left(\frac{e^{2}}{\kappa \ell}\right) \sum_{\mathbf{q}} X(\mathbf{q}) \mathbf{P}(-\mathbf{q}) \cdot \mathbf{P}(\mathbf{q})
\end{aligned}
$$

where

$$
\Upsilon(\mathbf{q})=2 H(\mathbf{q})-X(\mathbf{q})
$$

With the hamiltonian of Eq. (48), we obtain the equation of motion of the density operator

$$
i \hbar \frac{d \rho(\mathbf{k})}{d t}=[\rho(\mathbf{k}), H]
$$

which we linearize by writing $\rho(\mathbf{k}) \rightarrow\langle\rho(\mathbf{k})\rangle+\delta \rho(\mathbf{k})$ where the average is evaluated in the HFA. Keeping terms up to linear order in $\delta \rho(\mathbf{k})$, we find

$$
\begin{aligned}
\hbar \frac{d \delta \rho(\mathbf{k})}{d t}= & \left(\frac{e^{2}}{\kappa \ell}\right) \sum_{\mathbf{G}}[\Upsilon(\mathbf{G})-\Upsilon(\mathbf{k}-\mathbf{G})] \\
& \times \sin \left(\mathbf{k} \times \mathbf{G} \ell^{2} / 2\right)\langle\rho(\mathbf{G})\rangle \delta \rho(\mathbf{k}-\mathbf{G}) \\
& -4\left(\frac{e^{2}}{\kappa \ell}\right) \sum_{\mathbf{G}}[X(\mathbf{G})-X(\mathbf{k}-\mathbf{G})] \\
& \times \sin \left(\mathbf{k} \times \mathbf{G} \ell^{2} / 2\right)\langle\mathbf{P}(\mathbf{G})\rangle \cdot \delta \mathbf{P}(\mathbf{k}-\mathbf{G})
\end{aligned}
$$

This is the equation of motion of the density in the GRPA.

To find an expression for the current operator valid at small $\mathbf{k}$, we make the approximation

$$
\sin \left(\mathbf{k} \times \mathbf{G} \ell^{2} / 2\right) \approx \mathbf{k} \cdot\left(\mathbf{G} \times \widehat{\mathbf{z}} \ell^{2} / 2\right)
$$

and use the continuity equation

$$
\frac{d \delta \rho(\mathbf{k})}{d t}=\frac{i}{e} \mathbf{k} \cdot \mathbf{j}(\mathbf{k})
$$

We get in this way

$$
\begin{aligned}
\mathbf{j}(\mathbf{k})= & -\frac{i}{2}\left(\frac{e^{3}}{\hbar \kappa}\right) \sum_{\mathbf{G}}(\mathbf{G} \times \widehat{\mathbf{z}} \ell)[\Upsilon(\mathbf{k}+\mathbf{G})-\Upsilon(\mathbf{G})] \\
& \times\langle\rho(-\mathbf{G})\rangle \delta \rho(\mathbf{k}+\mathbf{G}) \\
& +2 i\left(\frac{e^{3}}{\hbar \kappa}\right) \sum_{\mathbf{G}}(\mathbf{G} \times \widehat{\mathbf{z}} \ell)[X(\mathbf{k}+\mathbf{G})-X(\mathbf{G})] \\
& \times\langle\mathbf{P}(-\mathbf{G})\rangle \cdot \delta \mathbf{P}(\mathbf{k}+\mathbf{G}) .
\end{aligned}
$$

This expression shows that the current can have contributions from both density and pseudospin fluctuations. In terms of the original $\rho_{i, j}(\mathbf{q})$ operators, we can write the current expression as

$$
\mathbf{j}(\mathbf{k})=-i \frac{e^{3}}{\hbar \kappa} \sum_{\mathbf{G}} \sum_{a, b} F_{a, b}(\mathbf{k}, \mathbf{G}) \delta \rho_{a, b}(\mathbf{k}+\mathbf{G}),
$$

where

$$
\begin{aligned}
& \mathbf{F}_{a, b}(\mathbf{k}, \mathbf{G}) \\
= & (\mathbf{G} \ell \times \widehat{\mathbf{z}})[H(\mathbf{k}+\mathbf{G})-H(\mathbf{G})]\langle\rho(-\mathbf{G})\rangle \delta_{a, b} \\
& -(\mathbf{G} \ell \times \widehat{\mathbf{z}})[X(\mathbf{k}+\mathbf{G})-X(\mathbf{G})]\left\langle\rho_{b, a}(-\mathbf{G})\right\rangle .
\end{aligned}
$$

With Eq. (55) for the current, we can easily write the current-current Matsubara Green's function tensor

$$
\chi^{J, J}(\mathbf{k}, \tau)=-N_{\varphi}\langle T \mathbf{j}(\mathbf{k}, \tau) \mathbf{j}(-\mathbf{k}, 0)\rangle,
$$

so that the retarded current-current response function is finally given by

$$
\begin{aligned}
\chi^{J, J}(\mathbf{k}, \omega)= & -\left(\frac{e^{3}}{\hbar \kappa}\right)^{2} \sum_{a, b, c, d} \sum_{\mathbf{G}, \mathbf{G}^{\prime}} \mathbf{F}_{a, b}(\mathbf{k}, \mathbf{G}) \\
& \times \chi_{n}^{a, b, c, d}\left(\mathbf{k}+\mathbf{G}, \mathbf{k}+\mathbf{G}^{\prime}, \omega\right) \mathbf{F}_{c, d}\left(-\mathbf{k},-\mathbf{G}^{\prime}\right)
\end{aligned}
$$

If we apply an external potential, the hamiltonian $H \rightarrow$ $H+H_{\text {ext }}$ with

$$
H_{e x t}=N_{\phi}\left(\frac{e^{2}}{\kappa \ell}\right) \sum_{a} \sum_{\mathbf{q}} W_{a}(-\mathbf{q}) \rho_{a, a}(\mathbf{q}) .
$$

We allow the potential $W_{a}(\mathbf{q})$ to be different for the $K$ and $K^{\prime}$ valleys. With this potential, the function $\mathbf{F}_{a, b}(\mathbf{k}, \mathbf{G})$ in Eq. (58) must be replaced by

$$
\begin{aligned}
& \mathbf{F}_{i, j}(\mathbf{k}, \mathbf{G}) \\
= & -(\mathbf{G} \times \widehat{\mathbf{z}} \ell) W_{K}(-\mathbf{G}) \delta_{i, j} \delta_{i, K} \\
& -(\mathbf{G} \times \widehat{\mathbf{z}} \ell) W_{K^{\prime}}(-\mathbf{G}) \delta_{i, j} \delta_{i, K^{\prime}} \\
& +(\mathbf{G} \ell \times \widehat{\mathbf{z}})[H(\mathbf{k}+\mathbf{G})-H(\mathbf{G})]\langle\rho(-\mathbf{G})\rangle \delta_{i, j} \\
& -(\mathbf{G} \ell \times \widehat{\mathbf{z}})[X(\mathbf{k}+\mathbf{G})-X(\mathbf{G})]\left\langle\rho_{j, i}(-\mathbf{G})\right\rangle .
\end{aligned}
$$

In pseudospin language, this means that the current in Eq. (54) becomes $\mathbf{j}(\mathbf{k}) \rightarrow \mathbf{j}(\mathbf{k})+\mathbf{j}_{W}(\mathbf{k})$, where

$$
\begin{aligned}
\mathbf{j}_{W}(\mathbf{k})= & -\frac{i}{4}\left(\frac{e^{3}}{\hbar \kappa}\right) \sum_{\mathbf{G}}(\mathbf{G} \times \widehat{\mathbf{z}} \ell) W_{+}(-\mathbf{G}) \delta \rho(\mathbf{k}+\mathbf{G} \oint 1) \\
& -\frac{i}{2}\left(\frac{e^{3}}{\hbar \kappa}\right) \sum_{\mathbf{G}}(\mathbf{G} \times \widehat{\mathbf{z}} \ell) W_{-}(-\mathbf{G}) \delta P_{z}(\mathbf{k}+\mathbf{G}),
\end{aligned}
$$


where $W_{ \pm}(-\mathbf{G})=W_{K}(-\mathbf{G}) \pm W_{K^{\prime}}(-\mathbf{G})$. Note that for consistency, we also include the external potential in the calculation of the $\left\langle\rho_{i, j}(\mathbf{G})\right\rangle^{\prime} s$ as well as in that of $\chi_{n}^{a, b, c, d}\left(\mathbf{k}+\mathbf{G}, \mathbf{k}+\mathbf{G}^{\prime}, \omega\right)$.

In the absence of any external potential, we find that, for the Wigner or meron crystal, only the phonon mode (and some higher energy modes) appears as a pole of $\Im\left[\chi_{x, x}^{J, J}(\mathbf{k}, \omega)\right]$ for any wave vector $\mathbf{k}$. The phase modes are conspicuously absent of the current response. We explain this by the fact that the phase modes are transverse modes: the motion of a pseudospin is perpendicular to the local value of that pseudospin so that the term $\left\langle\mathbf{P}\left(-\mathbf{G}^{\prime}\right)\right\rangle \cdot \delta \mathbf{P}\left(\mathbf{k}+\mathbf{G}^{\prime}\right)=0$ in Eq. (54). Also, these modes have no weight in the density-density response function. They cannot contribute to the local current.

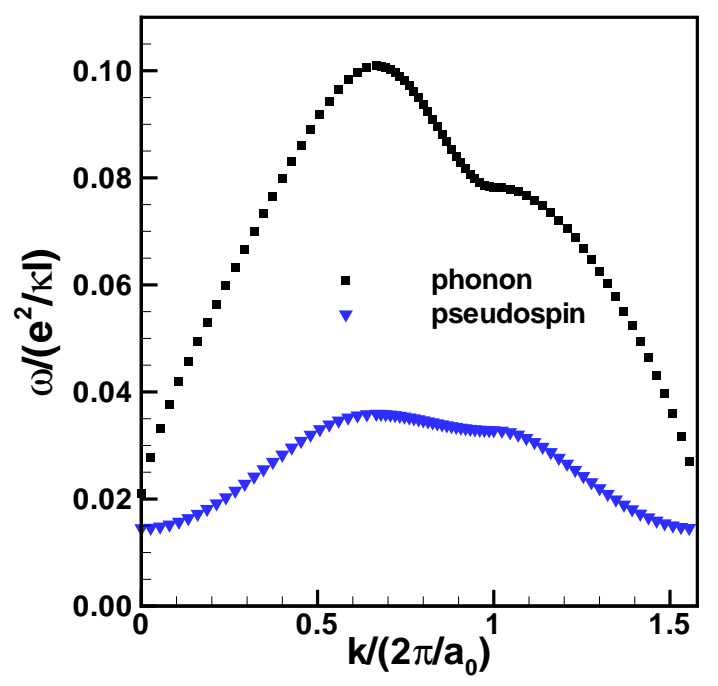

FIG. 10: Dispersion relation of the phonon and pseudospin modes of the Wigner crystal at $\nu_{0}=0.4$ in Landau level $n=0$ in an external potential $W_{K}=-0.005$ and $W_{K^{\prime}}=0$. The dispersion is plotted along the irreducible Brillouin zone of the triangular lattice (see Fig. 5). Both the phonon and pseudospin wave mode are gapped by the external field.

This conclusion is unchanged, for the Wigner crystal state, if we apply an external potential with $W_{K}(\mathbf{q})=$ $W_{K^{\prime}}(\mathbf{q})$. The phonon mode is gapped by that potential but the pseudospin mode dispersion remains gapless because the pseudospin symmetry has not been broken. To induce a gap in the pseudospin mode, we must allow $W_{K}(\mathbf{q}) \neq W_{K^{\prime}}(\mathbf{q})$ so that the external potential can couple to the $z$ component of the pseudospin: $P_{n, z}(\mathbf{q})=\left(\rho_{n}^{+,+}(\mathbf{q})-\rho_{n}^{-,},(\mathbf{q})\right) / 2$. We show in Fig. 10 the dispersion relation of the collective modes of the Wigner crystal for $\nu=0.4$ and with an external potential that is different in the two valleys i.e. $W_{K}=-0.005$ and $W_{K^{\prime}}=0$. Both the phase and phonon modes are now gapped as expected but Fig. 11 shows that, once again, only the phonon appears as pole of the current. This fact can be readily understood: the external potential is much weaker than the exchange energy that forces the parallel alignment of the pseudospins. It follows that when the external potential is applied, the pseudospins all align along the $z$ axis even though the external potential is modulated in space. The pseudospin mode is a transverse mode and so both terms $\left\langle\mathbf{P}\left(-\mathbf{G}^{\prime}\right)\right\rangle \cdot \delta \mathbf{P}\left(\mathbf{k}+\mathbf{G}^{\prime}\right)$ and $\delta P_{z}(\mathbf{k}+\mathbf{G})$ in the definition of the current in Eq. (54) are zero. For the phonon mode in the Wigner crystal state, the peak in the current response stays finite with decreasing wave vector so that the phonon mode is visible at $\mathbf{k}=0$ and can contribute to the absorption.

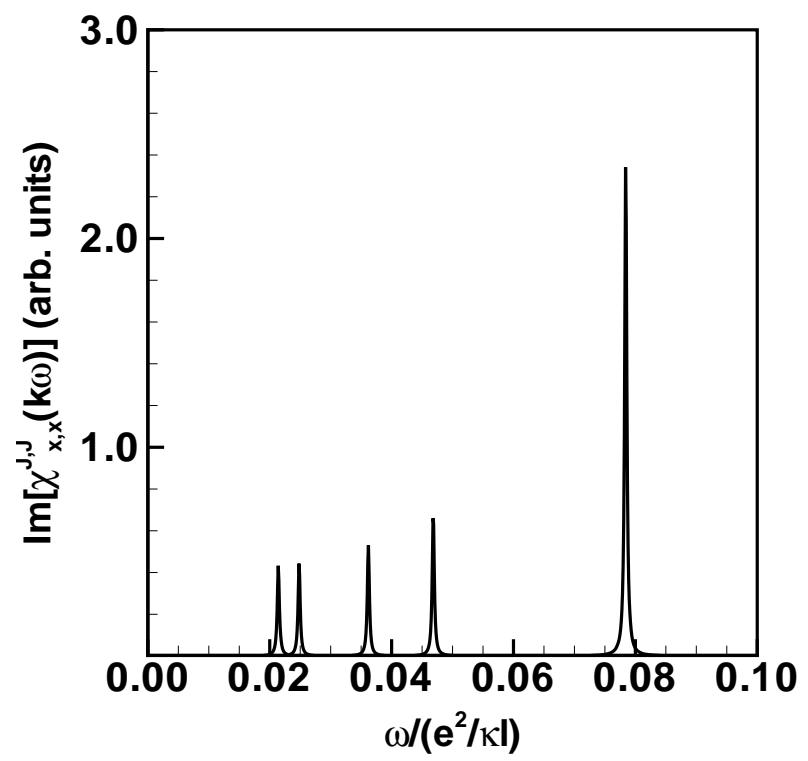

FIG. 11: Imaginary part of the current response function. From left to right: $k_{x}=k_{y}=0.001,0.01,0.05,0.1,0.3$ in units of $2 \pi / a_{0}$. These peaks come from the phonon mode. There is no peak coming from the pseudospin mode.

For the meron crystal, the situation is more complicated. An external potential $W_{-}(-\mathbf{G})$ acts as a pseudomagnetic field. If it is uniform in space, it acts effectively as a pseudospin Zeeman coupling and produces a transition from a meron to a bimeron crystal with two Goldstone modes (the phonon mode and a gapless pseudospin mode related to the $U(1)$ symmetry of the hamiltonian) and two gapped pseudospin modes ${ }^{16}$. The pseudospin modes being transverse and the second term in $\mathbf{j}_{W}(\mathbf{k})$ being zero because of the uniformity of the potential, there should again be no contribution of these modes to the conductivity. This is the case for two of the pseudospin modes, but the gapless pseudospin mode has a weight in the density-density response function at finite wave vector $\mathbf{k}$ and does appear in the current response along with the phonon peak. Both peaks go to zero with decreasing wave vector however.

For the meron crystal, the second term in $\mathbf{j}_{W}(\mathbf{k})$ 
should be finite if $W_{-}(-\mathbf{G})$ is non-uniform, in contrast with the Wigner crystal case, because some of the pseudospin modes involve a fluctuation in the $\hat{z}$ component of the pseudospin. Unfortunately, we find the meron crystal to be very sensitive to an inhomogeneous external potential, as indicated by instabilities in the collective mode spectrum at small values of $k$. This is likely related to the extreme closeness in energy of the meron crystal and meron pair crystal states, so that the external potential may lead to a different and possibly more complicated textured state. Thus it is not possible to compute the response functions at small wave vector $k$ for these textured states without better knowledge of their groundstate structure in the presence of a pinning potential. We note that at finite wavevector, where the dispersion is well behaved, the pseudospin modes do appear in the current response as expected.

In spite of the difficulty demonstrating the presence of a signature of the pinned phase modes in the dynamical conductivity at small wavevector, we believe at least a small response will in fact generically always be present. Beyond the density fluctuations in the pseudospin modes due to spin-charge coupling, there is a further density response due to the fact that the $A$ and $B$ sites of the lattice are at difference positions in real space in a unit cell. In our calculations, $A$ and $B$ were treated as two orthogonal "spin" states of an electron, but their slightly different locations in real space were not included in the model. If this were included, we expect that an oscillation of the pseudospin that changes the relative weight of an electron on the $A$ or $B$ sublattice (or, equivalently, on the $K$ and $K^{\prime}$ valleys) will translate into a change in the position of that electron or into a dipole fluctuation. Thus, if this distinction were properly included in our model, we would expect that the phase mode would appear as a pole of the current response just as the phonon mode does, albeit weakly, since the symmetry breaking is small.

In closing this section, we remark that, in Fig. 4, the effective stiffness for the pseudospin mode is two orders of magnitude smaller than that of the Wigner crystal for filling factor $\nu \lesssim 0.2$. In the presence of disorder, we might then expect two pinning modes of very different frequencies for a Wigner crystal and it may be impossible to detect the two modes simultaneously in an actual experiment. The meron crystal dispersion does not suffer from this problem since all four modes appear to have similar bandwidths.

\section{CONCLUSION}

We have shown in this work that the 2DEG in graphene can support Wigner crystals and meron crystals with valley-pseudospin textures. Our numerical analysis was restricted to filling factor $\nu_{n} \in[0.1,0.9]$ in each Landau level $n$ and we concluded that, in this range, meron crystals are present in Landau levels $n=0$ and $n=1$ only. We have computed the dispersion relation of the collective excitations of these two crystal states and showed that the Wigner crystal has one extra Goldstone mode with a quadratic dispersion at small wave vector in addition to the phonon mode. Meron crystals have 3 extra Goldstone modes in addition to the phonon mode. These extra Goldstone modes are valley-pseudospin fluctuations. In graphene, all these modes involve density fluctuations, and we speculated that these last modes could be visible as pinning modes in microwave absorption spectrum in a real disordered system.

\section{Acknowledgments}

This work was supported by a research grant from the Natural Sciences and Engineering Research Council of Canada (NSERC) for R. Côté and an NSF Grant No. DMR-0704033 for H. A. Fertig. Computer time was provided by the Réseau Québécois de Calcul Haute Performance (RQCHP).
1 P. K. Lam and S. M. Girvin,Phys. Rev. B 30, 473 (1984); D. Levesque, J. J. Weis, and A. H. MacDonald, Phys. Rev. B 30, 1056 (1984); K. Esfarjani and S. T. Chui, Phys. Rev. B 42, 10758 (1990); K. Yang, F. D. M. Haldane, and E. H. Rezayi, Phys. Rev. B 64, 081301(R) (2001); X. Zhu and S. G. Louie, Phys. Rev. B 52, 5863 (1995).

2 For recent reviews, see Physics of the electron solid, edited by S. T. Chui (International Press, Boston (1994) and H. Fertig and H. Shayegan in Perspectives in Quantum Hall Effects, edited by S. Das Sarma and A. Pinczuk (Wiley, New York, 1997), Chaps. 5 and 9 respectively.

3 P. D. Ye, L. W. Engel, D. C. Tsui, R. M. Lewis, L. N. Pfeiffer, and K. West, Phys. Rev. Lett. 89, 176802 (2002); Yong P. Chen, G. Sambandamurthy, Z. H. Wang, R. M. Lewis, L. W. Engel, D. C. Tsui, P. D. Ye, L. N. Pfeiffer, and K. W. West, Nat. Phys. 2, 452 (2006); Y. P. Chen, R.
M. Lewis, L. W. Engel, D. C. Tsui, P. D. Ye, L. N. Pfeiffer, and K. W. West, Phys. Rev. Lett. 91, 016801 (2003); R. M. Lewis, Yong Chen, L. W. Engel, D. C. Tsui, P. D. Ye, L. N. Pfeiffer, and K. W. West, Physica E22, 104 (2004); R. M. Lewis, P. D. Ye, L. W. Engel, D. C. Tsui, L. N. Pfeiffer, and K. W. West, Phys. Rev. Lett. 89, 136804 (2002); R. M. Lewis, Y. Chen, L. W. Engel, D. C. Tsui, P. D. Ye, L. N. Pfeiffer, and K. W. West Phys. Rev. Lett. 93, 176808 (2004); R. M. Lewis, Yong Chen, L. W. Engel, P. D. Ye, D. C. Tsui, L. N. Pfeiffer, and K. W. West, Physica E22, 119 (2004); Yong P. Chen, R. M. Lewis, L. W. Engel, D. C. Tsui, P. D. Ye, Z. H. Wang, L. N. Pfeiffer, and K. W. West, Phys. Rev. Lett. 93, 206805, 2004.

4 A. A. Koulakov, M. M. Fogler and B. I. Shklovskii, Phys. Rev. Lett. 76, 499 (1996); M. M. Fogler, A. A. Koulakov, and B. I. Shklovskii, Phys. Rev. B 54, 1853 (1996); R. 
Moessner and J.T. Chalker, Phys. Rev. B 54, 5006 (1996); M. M. Fogler and A. A. Koulakov, Phys. Rev. B 55, 9326 (1997). For a review of the bubble and stripe phases in higher Landau levels, see M. Fogler in High Magnetic Fields: Applications in Condensed Matter Physics and Spectroscopy, ed. by C. Berthier, L.-P. Levy, G. Martinez (Springer-Verlag, Berlin), 99 (2002).

5 R. Côté, C. B. Doiron, J. Bourassa, and H. A. Fertig, Phys. Rev. B 68, 155327 (2003).

6 C.-H. Zhang and Yogesh N. Joglekar, Phys. Rev. B 75, 245414 (2007).

7 C.-H. Zhang and Y. N. Joglekar, Phys. Rev. B 77, 205426 (2008).

8 M. I Katsnelson, K. S. Novoselov, and A. K. Geim, Nature Physics 2, 620 (2006).

9 J. Wang, H.A. Fertig, A.P. Iyengar, and L. Brey, unpublished.

10 M. O. Goerbig, R. Mosessner, and B. Douçot, Phys. Rev. B 74, 161407(R) (2006).

11 K. Yang, S. Das Sarma, and A. H. MacDonald, Phys. Rev. B 74, 075423 (2006).

12 L. Brey, H.A. Fertig, R. Côté, and A.H. MacDonald, Physica Scripta, T 66, 154 (1996).

13 A. H. Castro Neto, F. Guinea, N. M. R. Peres, K. S. Novoselov, and A. K. Geim, arXiv:0709.1163 (To be published in Review Modern Physics (2008)).
14 J. Bourassa, B. Roostaei, R. Côté, H. A. Fertig, and K. Mullen, Phys. Rev. B 74, 195320 (2006).

${ }^{15} \mathrm{~W}$. Luo and R. Côté, unpublished.

16 R. Côté, D. B. Boisvert, J. Bourassa, M. Boissonneault, and H. A. Fertig, Phys. Rev. B 76, 125320 (2007).

17 R. Côté and A. H. MacDonald, Phys. Rev. B 44, 8759 (1991); Phys. Rev. Lett. 65, 2662 (1990).

18 A.P. Iyengar, J. Wang, H.A. Fertig, and L. Brey, Phys. Rev. B 75, 125430 (2007).

19 Y.V. Nazarov and A.V. Khaetskii, Phys. Rev. Lett. 80, 576 (1998).

20 R. Chitra, T. Giamarchi, and P. Le Doussal, Phys. Rev. B65, 035312 (2001); ibid., Phys. Rev. Lett. 80, 3827 (1998).

21 H. A. Fertig, Phys. Rev. 59, 2120 (1999).

22 R. Côté, Mei-Rong Li, A. Faribault, and H. A. Fertig, Phys. Rev. B 72, 115344 (2005).

23 S. M. Girvin, A. H. MacDonald, and P. M. Platzman, Phys. Rev. B 33, 2481 (1986).

24 J. Martinez and M. Stone, International Journal of Modern Physics B 7, 4389-4401 (1993); R. Rajaraman, International Journal of Modern Physics B 8, 777-788 (1994); R. Rajaraman and S. L. Sondhi, Modern Physics Letters B 8, 1065-1073 (1994). 